\title{
VIRTUAL CONCEPTUAL DESIGN AND ANALYSIS OF MULTIFUNCTIONAL ROBOT
}

\author{
Ramesh Kumar K R, ${ }^{1}$ Prasad Krishna ${ }^{1}$, Nagarathan H.S ${ }^{2}$ \\ ${ }^{I}$ Department of Mechanical Engineering National Institute of Technology Karnataka, Surathkal, Karnataka, India \\ ${ }^{2}$ Department of Electronics \& Communication Engineering, Dr.Ambedkar Institute of Technology Bangaluru, \\ Karnataka, India
}

\begin{abstract}
In the actual world, any unremitting medium deforms under the application of force. Rigid body simulations do not confine such deformations and may lead to inexact results. As a virtual prototype replaces physical prototype, tests can be carried out to validate the design of the product. Virtual prototype of multifunctional robot is developed to simulate the system level working with live stress distribution, which is designed to lift a specific payload. In this work, a miniature Computer Control Robotic Vehicle $(C C R V)$ with five degrees of freedom (5DOF) robotic arm has been designed and developed. The main objective of this article is to design and analysis of Robot manipulator is to lift $500 \mathrm{gm}$ Payload is divided into two parts. In this paper design and analysis will rely heavily on design and analysis tool, namely CATIA V5R19 and NASTRAN/PATRAN.
\end{abstract}

Keywords- Simulation, Virtual Prototype, Payload, Analysis, CATIA, MBD.

\section{INTRODUCTION}

The multifunctional robot consists of several parts, namely base frame, vertical column, horizontal column, end effector, gripper and wheels. The design and drawing of a multifunctional robot are carried out in the software called CATIA V5R20 (Computer Aided Three-Dimensional Interactive Application). Inclusion of flexible bodies in MBD (Model Based Design) simulations accounts for flexibility. [10] Model based methodologies have become the basis of study on implanted systems growth. The availability of established computer aided tools and of wellsettled industrial practices have promoted the implementation of these methodologies in large companies, which are able to pay back the cost on a large volume of products. Methodology of MBD here individual parts of a multifunctional robot is designed with the suitable dimensions. Once all the parts of a multifunctional robot are developed in CATIA then it will be assembled and simulated. As a virtual prototype replaces physical prototype, tests can be carried out to validate the design of the product. Virtual prototype of multifunctional robot is developed with a Flexi body concept to simulate the system level working with live stress distribution, which is designed to lift a design payload of $500 \mathrm{gm}$. The robot arm is converted into Flexi body as it is a critical component to validate the design of robot manipulator. The previous work that has been done to gain more information about literature. The beginning part of this paper explains the history of Industrial Robots and types of robots that are available. This will be followed by information pertaining to robot grippers or end effectors. Finally, information on robot manipulator design consideration will be available at the end.

Rujeko music \& Jaydeep Roy et al [1]-[12] carried out the Finite element analysis, they have showed FEA is a emerging technique used to obtain expected solutions of boundary value problems in engineering. Boundary value problems are also called field problems. The field is the domain of interest and most often represents a physical structure, i.e. the robot manipulator.M.H Hojjati et al showed that [2] in a conventional finite element analysis, material properties, dimensions and applied loads are usually defined as deterministic quantities. This simplifying assumption, however, is not true in practical applications. Xuewei Zhang uses three-dexel modeling method [3] to developed system, it is validated experimentally to show that the modeling, simulation, and optimization methods could improve the accuracy and efficiency of five-axis milling processes. The flexible mechanical structure as a system with slow (rigid) and fast (flexible) modes that can be controlled separately [4]-[13] the flexible link robotic manipulators have many advantages with respect to conventional rigid robots. These mechanisms are built using lighter, cheaper materials, which improve the payload to arm weight ratio, thus resulting in an increase of the speed with lower energy consumption. Further Ikuo Mizuuchi, Ryosuke Tajima in 2002 developed geometric virtual robot model he showed that [5] changeable flexibility of a structure is somewhat different from the adjustable flexibility of a joint. In the case of flexible joint, the ability to absorb shock is often diminished by the large mass of the descendant links, compared with flexible structure. R. Iglesias et al conducted experiments with a Magellan Pro mobile robot they demonstrate that [6] Machine-learning techniques, such as artificial neural networks commonly used to obtain the desired sensor-motor competencies. However, although these methods speed up the development of a reactive controller significantly, most of them produce opaque models that cannot be used to investigate and understand the characteristics of the robot's behavior further. The major development of a flexure-based parallel manipulator (FPM) [7]-[14] inspite such research efforts, it 
is still not easy issue to develop multi-DOF nanopositioning FPMs with large workspace and high payload (e.g., more than $100 N$ ). J. Jesus Cervantes-Sanchez et al [8] presented a comprehensive approach for the static analysis of spatial parallel manipulators using the principle of virtual work, the principle of virtual work is a very powerful tool since the reactions and internal forces are not involved in the formulation, and the number of equations is greatly reduced. Pietro Buttolo and Blake Hannaford [15] describe the structure of a force display recently implemented for precision manipulation of scaled or virtual environments. Hee-Seong Park, Chang-Hwan Choi 2009 carried out case studies [9] to find out if a virtual manipulator by using a 6 DOF haptic device could access a failed part of pyroprocessmg devices, and if a human operator could feel the force feedback when it collides with other objects when accessing a device.

From the available literature it is clearly shown that design and development of multifunctional robot in a virtual environment are limited and few reports have been made. Present work we focus our attention how virtual conceptual design and development of multifunctional robot in rigid and flexible body concept to shows live stress distribution.

\section{METHODOLOGY}

Procedure fallowed in this work is listed below:

- Requirement collection of the robot

- Design/Drawing

- Modelling of individual parts using CATIA CAD tool

- Assembly of all the parts

- Analysis
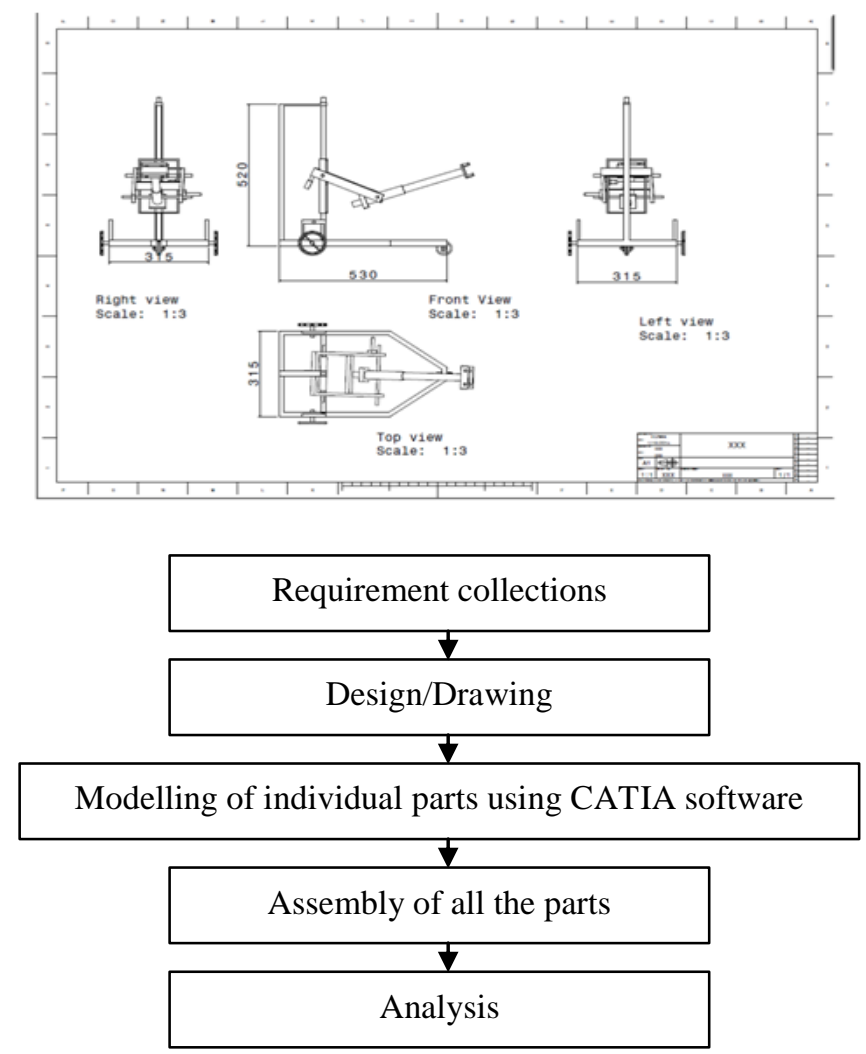

Fig 1. Base Frame

\subsection{Methodology}

\subsection{Requirement Collection of the Robot}

- Number of axes.

- Degrees of freedom.

- Working envelope.

- Kinematics.

- Carrying capacity or payload.

- Speed.

- Acceleration

- Accuracy.

- Repeatability.

\subsection{Design and Drawingco}

Computer programs using computer-aided design systems were employed to aid in the design of the manipulator arm [11] the mechanical design of the manipulator is aimed to withstand specified payload and a high stiffness-to-weight ratio of the links.

The two dimensional drawing and three dimensional CAD models of multifunctional robot is drawn using CATIA software. Design of all the parts such as Drawing of Base with L column, CAD model of Base with L column, Drawing of the vertical column, CAD model of Vertical column, Drawing of Gripper, CAD model of Gripper, Drawing of Link assembly, CAD model of link assembly, Assembly of Multifunctional robot and virtual prototyping of robot are designed as shown in the figure 2.2.

Fig 2.2 Two dimensionl view of a robot

\subsection{Modelling And Assembly Of Robot}

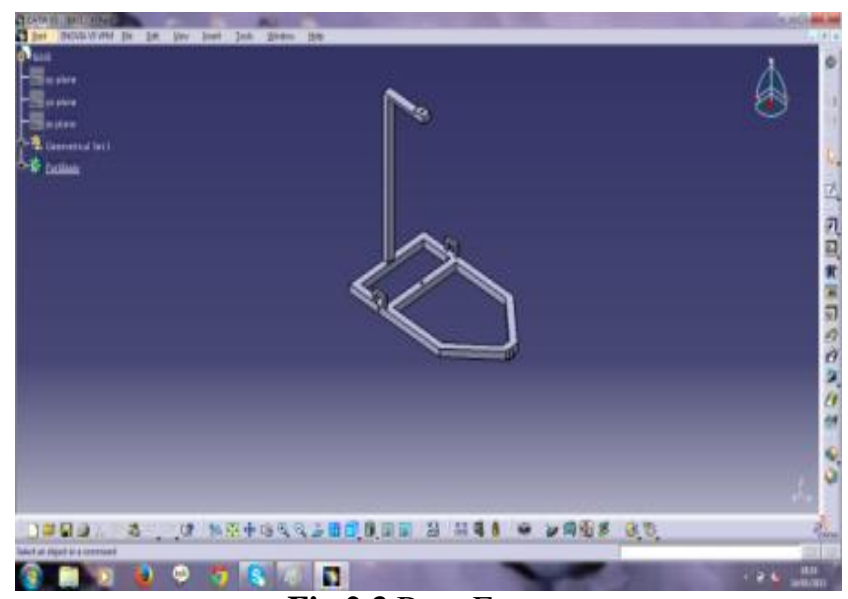

Fig 2.3 Base Frame

Base frame has to be stronger. So we have designed this Base frame which will act as a support between vertical column and horizontal column. One end of the Base frame is being connected to the vertical column and the other end is connected to the base of the Base frame. Provision is being given in the Base frame such as slots are provided to fix the vertical column in these slots. And also the Base frame has got three wheels to be fitted, one at the front and the other two at the rear end which will make the robot to move forward and backward as shown in fig 2.3. 


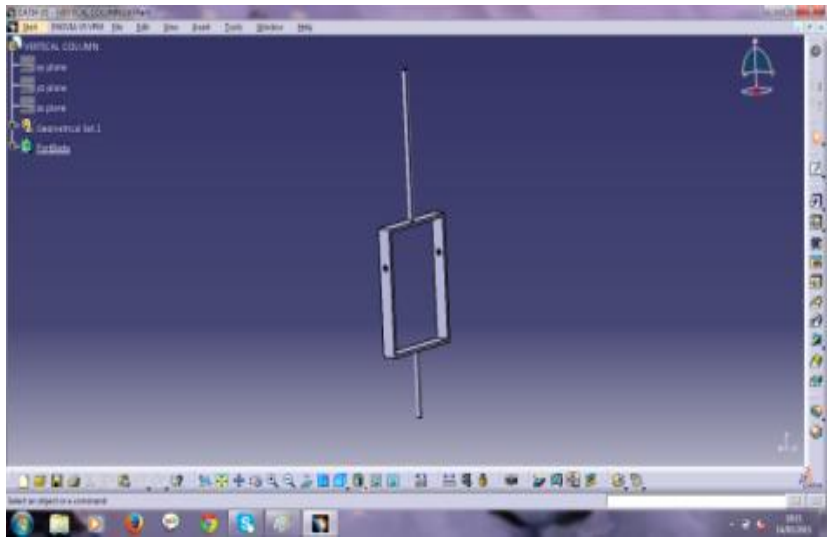

Fig 2.4 Vertical Column

Fig 2.4 shows the $3 \mathrm{D}$ view of the vertical column. The vertical column is attached to one end of the Base frame and the other end is being connected to topmost supporting column of the robot. The vertical column can be moved according to the degrees of freedom which we input.

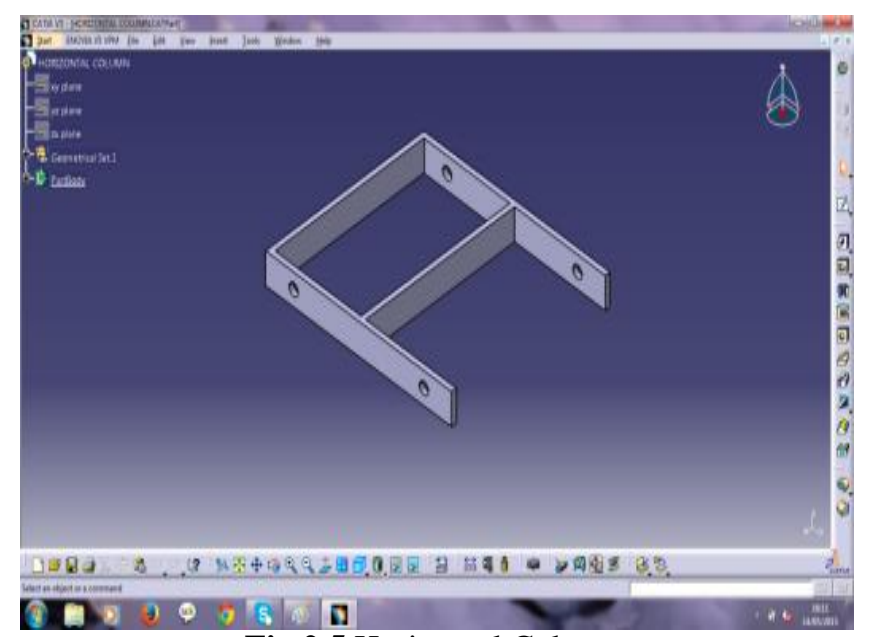

Fig 2.5 Horizontal Column

The 3D view of the horizontal column. The horizontal column is an interface between the vertical column and end effector arm as shown in fig 2.5.

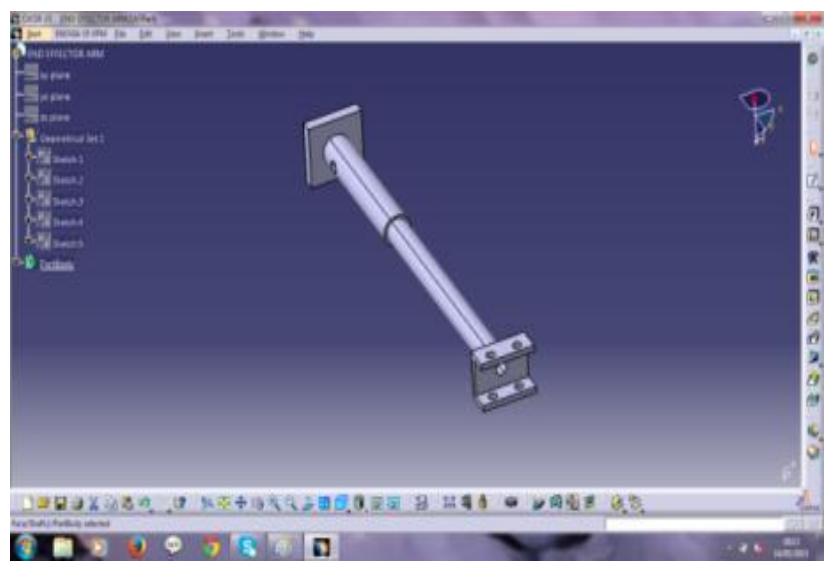

Fig 2.6 End Effector Arm

Fig 2.6 shows end effector arm, the design of the end effector arm is suitable to pick a load of $500 \mathrm{gm}$.

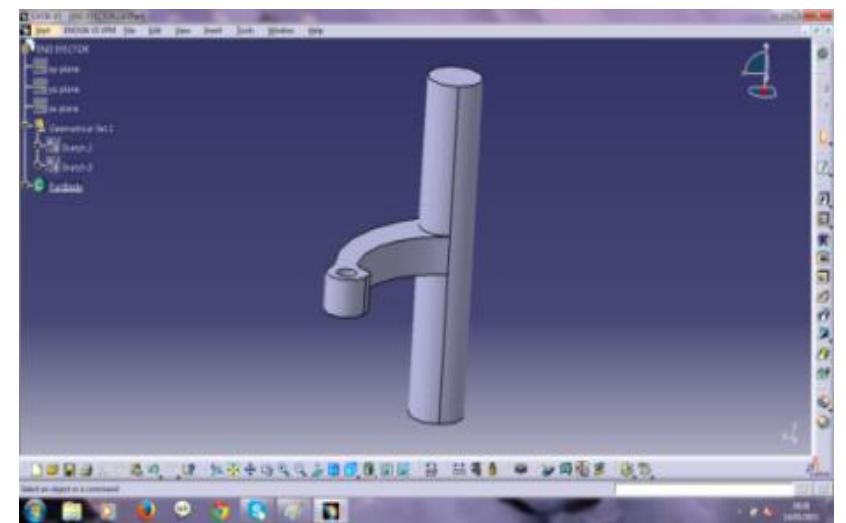

Fig 2.7 Gripper

Fig 2.7 shows 3D view of the grippers,called as the main components of the robot. The grippers are those which holds the material by opening and closing. The grippers does the mechanism of opening and closing, which helps the gripper to hold the material.

The robotic model consists of three wheels one at the front and the other two at the rear end. The front wheel will be freely moved. The front wheel is fixed to the Base frame and the provisions are provided to fix the wheels for it.

The robotic model consists of three wheels one at the front and the other two at the rear end. The front wheel will be freely moved as shown in fig 2.8. The front wheel is fixed to the base frame and the provisions are provided to fix the wheels to it.

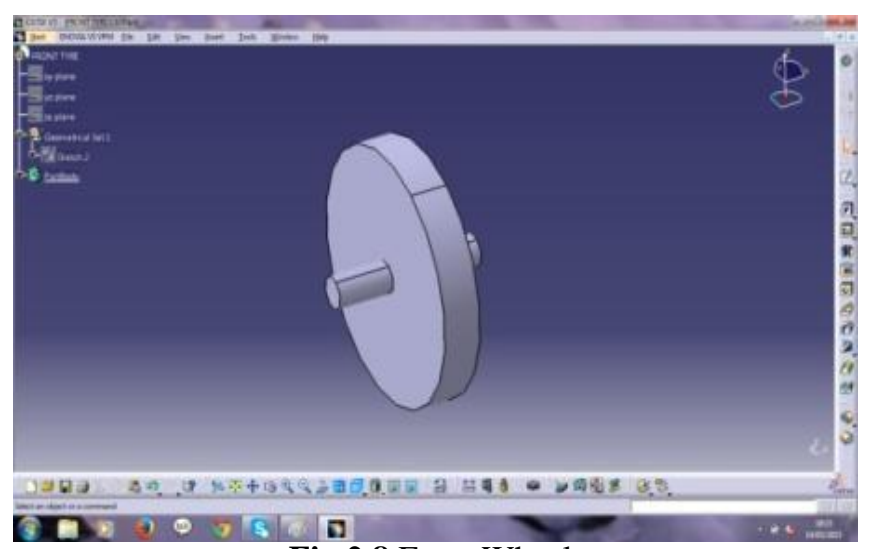

Fig 2.8 Front Wheel

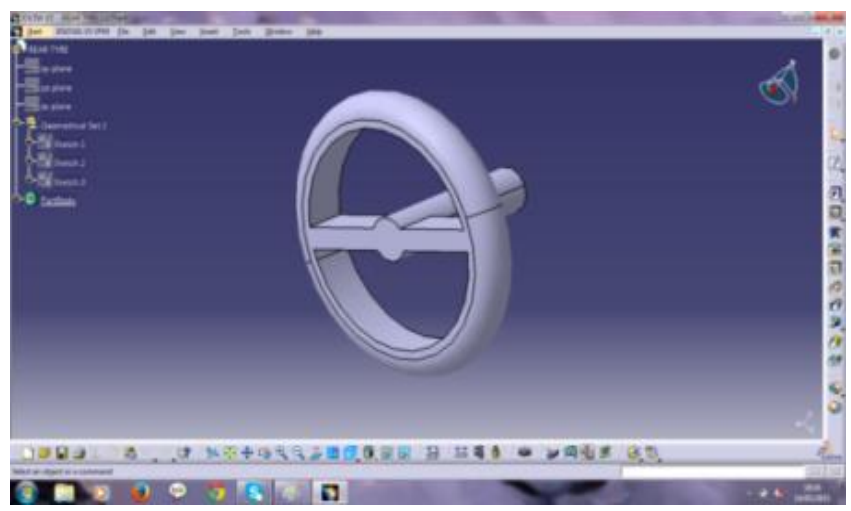

Fig 2.9 Rear Wheel 
There are two rear wheels in this robotic model. The rear wheels are fixed to the Base frame. Fig 2.9 shows the One of the rear wheel of the robot.

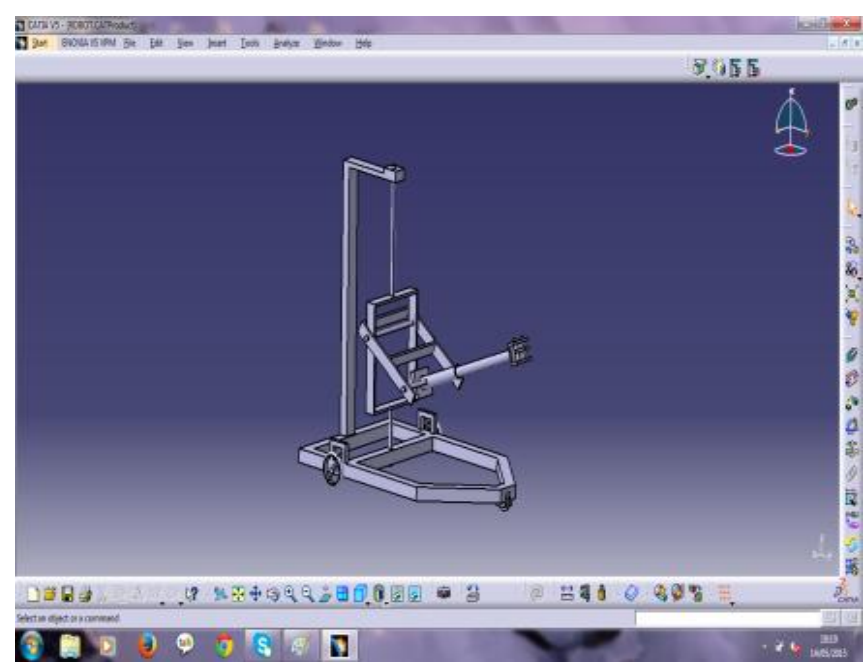

Fig 2.10 Assembly

The assembly of the robot model is being shown in the figure 2.10. Once all the parts of the robot model are being designed and drawn, then we need to assemble it so that to form a robotic unit.

\section{ANALYSIS}

\subsection{Finite Element Method}

Finite element method, sometimes referred as finite element analysis, is a computational technique used to obtain approximate solutions of boundary value problems in engineering. Boundary value problem is mathematical problems in which one or more dependent variables must satisfy specific conditions the boundary of the domain. Boundary value problems are sometimes called field problems. The field is the domain of interest and most often represents a physical structure.

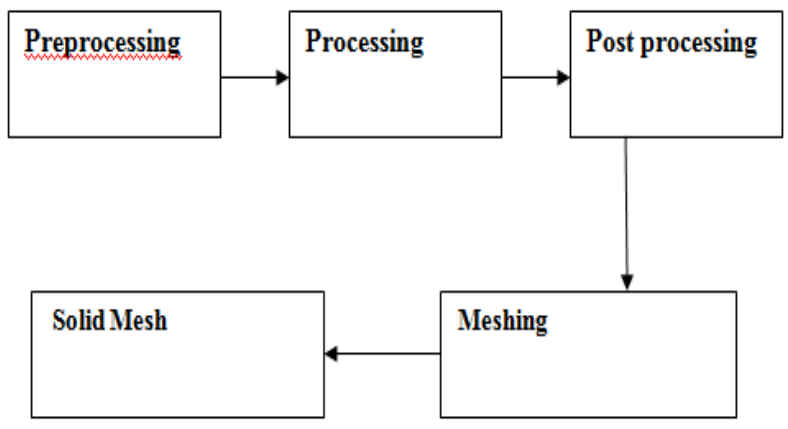

Fig 3.1 Procedure of Finite Element Analysis

\subsection{Pre-processing}

- Conversion of geometric model into finite element model.

- Material property data representation.

- Defining the boundary and loading conditions.

\subsection{Processing}

- Solve the system of linear algebraic equation.

- Stresses are determined from the strains by hook's law.

- Strains are derived from the displacement functions within the element combined with

- Hooke's law.

\subsection{Post Processing}

- Output from finite element analysis in numerical form.

- It usually consists of nodal values of the field variables and its derivatives.

\subsection{Meshing}

A mesh is a network of line elements and interconnecting nodes used to model a structural system and numerically solve for its simulated behavior under applied loading. Different element, size can select for different component cases.

\subsection{Solid Mesh}

Solid mesh is used for two dimensional components, when a dimension of the two directions is greater than the third direction than shell mesh can be used. Generally thick and thin components selected for solid meshing.

\subsection{Nastran}

Nastran is software which is used for finite element analysis and computer aided engineering. It was invented by NASA. Nastran is used in the automotive, aerospace, and industrial products industries. Nastran also provides a good collection of multi-physics capabilities, such as coupled acousticstructural, piezoelectric, and structural-pore capabilities, making it attractive for production-level simulations where multiple fields need to be coupled.

\subsection{Linear Static Analysis}

In linear static analysis displacements, strains, stresses, and reaction forces under the effect of applied loads are calculated. A series of assumptions are made with respect to a linear static analysis:

- Small deflections

- Material properties

- Constant boundary conditions

\subsection{Meshing Of Robot for Linear Static Analysis}

Meshed model is done by using tetra mesh. In tetra mesh the thickness of the component is to be mentioned, so the thickness of Robot is $1.6 \mathrm{~mm}$ of steel. Static is the study of structures at a fixed point in time and dynamics is the study of structures over a period of time. Basically statics study things that don't move, while dynamics studies things that do. Statics is concerned with moments, forces, stresses, torque, pressure, etc. Dynamics are concerned with displacement, velocity, acceleration, momentum, etc. Statics is concerned about how a mechanical system would act if everything is perfectly motionless and rigid 


\subsection{Material Properties of ROBOT}

Here we assumed steel and composite materials are isotropic in nature

Table 3.1 Material properties

\begin{tabular}{|l|l|}
\hline Property & Mild steel \\
\hline Density $\left(\mathrm{Kg} / \mathrm{m}^{3}\right)$ & 7850 \\
\hline Modulus of elasticity $(\mathrm{MPa})$ & $2 \times 10^{5}$ \\
\hline Poisson ratio & 0.3 \\
\hline Tensile strength $(\mathrm{MPa})$ & 460 \\
\hline Yield strength $(\mathrm{Mpa})$ & 250 \\
\hline
\end{tabular}

\section{RESULT AND DISCUSSIONS}

\subsection{Procedure for Analysis Using PATRAN}

\section{Software}

Here in this project we have considered the weight of the motor, the weight of the individual parts of robot and also counter weight. A node is created just above the robotic model and all the weights are added through the node on the desired parts of the robot model as shown in the fig Here the weights of different parts of robotic model are listed below,

- Weight of the gripper - $187 \mathrm{gm}$

- Weight of the end effector arm $-1.5 \mathrm{~kg}$

- Weight of the vertical column $-1.9 \mathrm{~kg}$

- Counter weight $-8 \mathrm{~kg}$

In order to avoid the end effector arm free fall when the gripper picks up a load of $500 \mathrm{gm}$ we need to add the counter weight on the other side which is fixed at the back end of horizontal column. The counter weight acts as a balancing the end effector arm from a free fall.

Considering all the weights than we import the files to PATRAN and NASTRAN software First, we develop the model in CATIA software. We have save it in the IGES format. This file will be imported to PATRAN software for Finite Element analysis. In Finite element analysis we can perform meshing, apply loads, material properties, boundary conditions, add counter weight and motor weight. Once it is being done, then the BDF file will be transferred to the NASTRAN software for getting the results. The results include files such as FO6, FO4, XDB, LOG which are read only file again sent back to PATRAN where we can get the results such as vonmises stress, deformation, axial stress and bending stress.

\subsection{NASTRAN Software Files}

When we run the BDF files in NASTRAN we get the results to read in four different files, namely, robot. f04, robot. f06, robot. $\mathrm{xdb}$ and robot.log.

Robot.log files fetch the information about how much memory the robotic model occupies, timings of execution and system performance etc as shown in the following figures.

\subsection{Tetrahedron Meshing}

For volume meshing, a tetrahedral mesh generally provides a more automatic solution with the ability to add mesh controls to improve the accuracy in arms critical region. Structural vibration frequencies of robot may vary significantly with arm orientation, payload, and mechanical boundary condition. Absent careful qualification of arm configuration payload, and boundary conditions.

Every model has 3 translational and 3 rotational degrees of freedom. Here 3 translational motion is with respect to $\mathrm{x}, \mathrm{y}$ and $\mathrm{z}$ and also 3 rotational motion with respect to RX, RY and $\mathrm{RZ}$ which needed to be fixed for meshing as shown in figure

\subsection{Application of Load}

Here we can input the weights and counter weight load and also can mention the boundary conditions on various parts of a robotic model as shown in figure 4.1. Here $x=0, y=0$ and $\mathrm{z}=-4.99 \mathrm{~N}$ because the force applied on the gripper part is acting downward which is in ' $z$ ' direction.

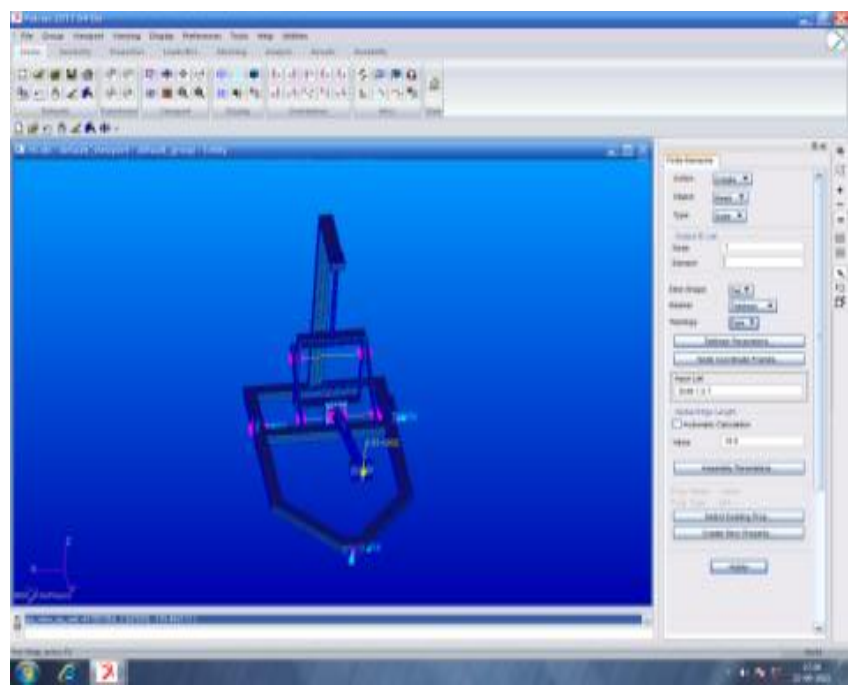

Fig 4.1 Tetmesh

\subsection{Material Properties}

In this we can assign the youngs modulus value, Poisson ratio and also the density of steel. Here we have assumed the value of youngs modulus as $2.5 \mathrm{E}^{-5} \mathrm{~N} / \mathrm{MM}^{2}$ and Poisson ratio as 0.33 and densities of steel as $7850 \mathrm{Kg} / \mathrm{m}^{3}$

\subsection{MPC (Multipoint Constraints)}

Multiple point constraints establish a relationship between degrees of freedom in one or more nodes, with the help of MPC we can create a node and connect all the weights of robots of different parts onto that single node. In between MPC we have connected bar element.

\subsection{Steps For Applying Load And Analysis}

Different loads ie $4.99 \mathrm{~N}$ in $\mathrm{z}$ direction because the force applied on the gripper part acting downward which is in the $\mathrm{z}$ direction. Separately applied mmotor weight and counter 
weight as shown in fig $4.2 \& 4.3$. After applying loads we can observe deformation on either side of the robot. Once we applied load, live stress distribution have seen in bit distribution file.

For the results we need to have the BDF file (as shown in fig 4.4) from the linear static model of the robot. Once we get the BDF file from this software we can send this file to NASTRAN software for getting the results. The results are obtained and fetched in read only format which can be read easily.

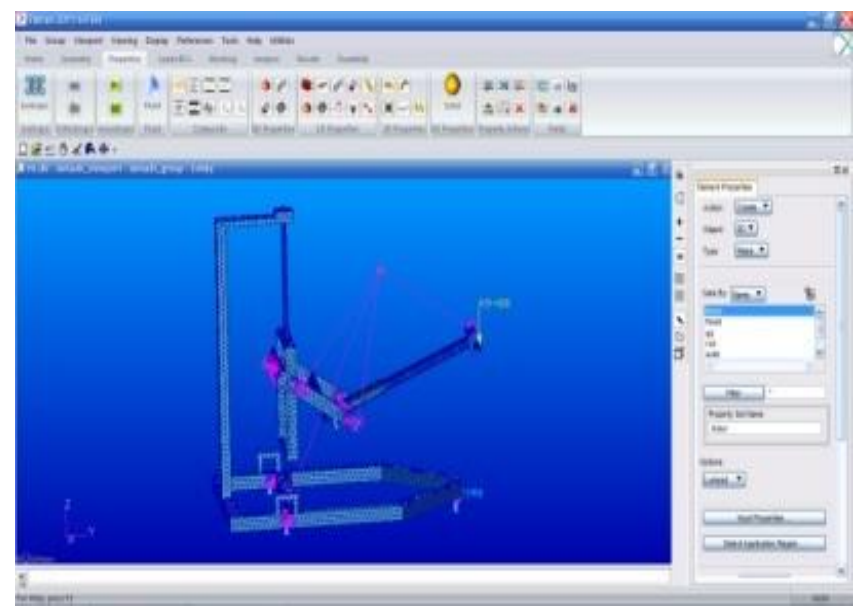

Fig 4.2 Counter Weight and Motor Weight

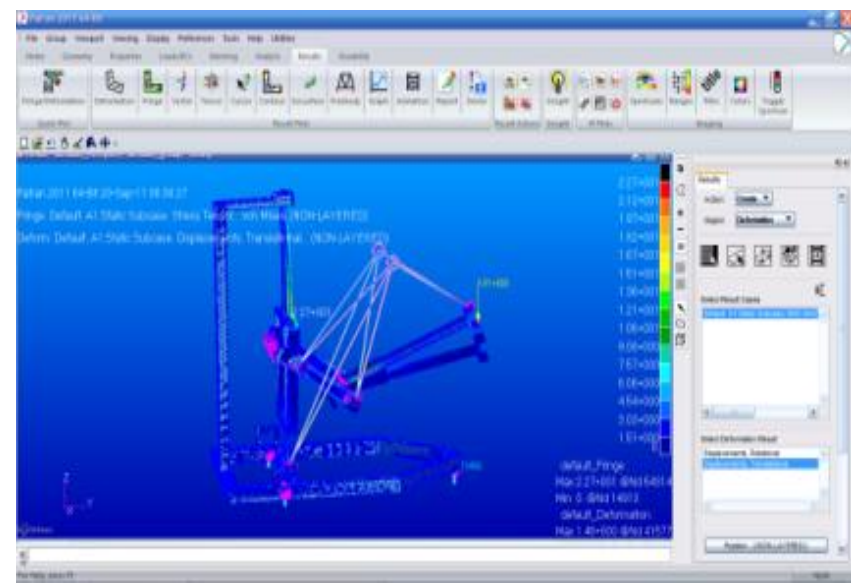

Fig 4.3 Deformation

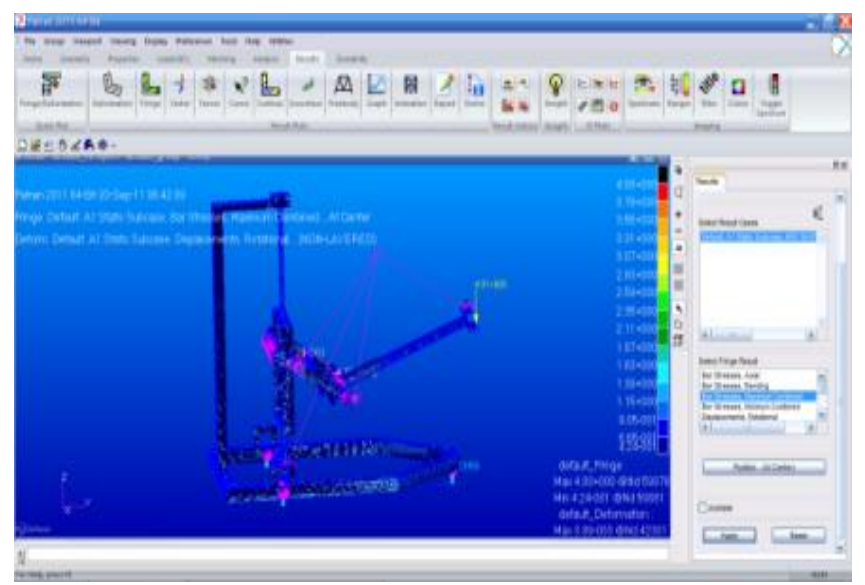

Fig 4.4 BDF File Creation

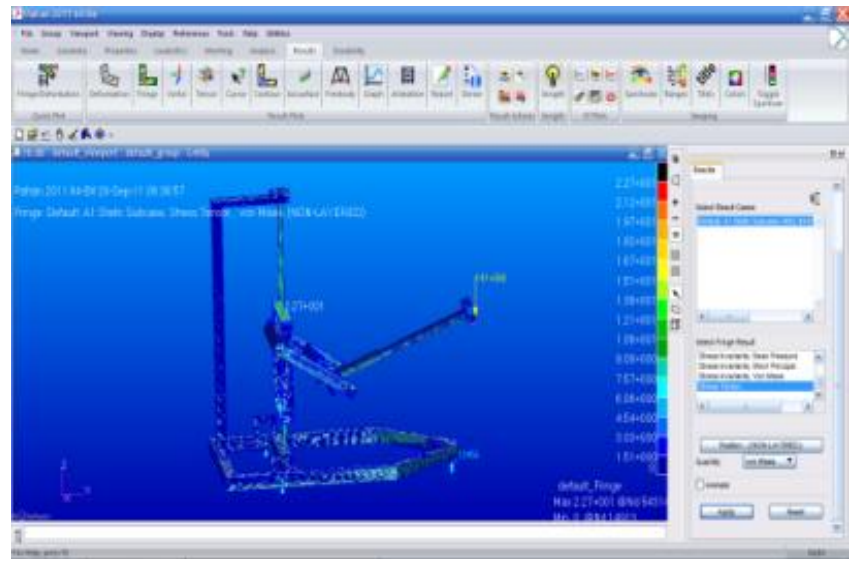

Fig 4.5 Vonmises Stress

\section{CONCLUSION}

It is clear from the above examination design and analysis have been done successfully to analyse live stress distribution of strength to weight ratio of a robot

- Rigid and flexible body concept we developed a multifunctional robot in an virtual environment for the real-time control of live stress distribution. This is very much necessary for the study of manipulators with multiple links and joints that are all flexible.

- The virtual Prototype of multifunctional robot is developed with rigid and flexi-body to visualize the working of multifunctional robot and to simulate the live stress distribution of robot arm which is design to lift a designed pay load.

- The general model formulation can be obtain the closedform dynamic models for practical flexible manipulators with any number of links.

- Analysis of the robot model is done using PATRAN and NASTRAN analysis tool. The results which are shown here is about mainly vonmises stress, which is found that 22.7 N/MM ${ }^{2}$ which is less than yield stress of steel. The robot can be fabricated as per the design and drawing.

\section{REFERENCES}

[1]. Rujeko Masike, Talon Garikayi, and Godfrey Tigere (2014) : Conceptual Design Framework for Developing a Cylindrical Robot Manipulator for Material Handling in Zimbabwean Small to Medium Enterprises: A Finite Element Approach International Journal of Advances in Mechanical and Automobile Engineering (IJAMAE) Volume 1, Issue 1

[2]. M.H Hojjati, A. Sadighi, (2009): Reliability Based Finite Element Analysis of Mechanical Components, Multidiscipline Modeling in Materials and Structures, Vol. 5 , pp. $151-162$.

[3]. Xuewei Zhang,Tianbiao Yu ,Wanshan Wang (2014): Modeling, simulation, and optimization of five-axis milling processes, Int J Adv Manuf Technol 74:16111624.

[4]. Victor Etxebarria, Arantza Sanz \& Ibone Lizarraga(2005): Control of a Lightweight Flexible Robotic Arm Using Sliding Modes, International 
Journal of Advanced Robotic Systems, Volume 2 pp. 103 110,Number 2, ISSN 1729-8806.

[5]. Ikuo Mizuuchi, Ryosuke Tajima, Tomoaki Yoshikai, Daisuke Sato, Koichi Nagashima, Masayuki Inaba, Yasuo Kuniyoshi, and Hirochika Inoue,(2002): The Design and Control of the Flexible Spine of a Fully Tendon-Driven Humanoid "Kenta", Proceedings of the IEEE/RSJ Int, Conference on Intelegent robots and system EPFL,lausanne,switzerland.

[6]. R.Iglesias b,U. Nehmzowa, S.A.Billings c (2008): Model identification and model analysis in robot training, International Journal of Robotics and Autonomous Systems Vol 56, pp 1061-1067.

[7]. Guilin Yang, Tat Joo Teo, I-Ming Chen,Wei Lin (2011): Analysis and Design of a 3-DOF Flexure-based Zero-torsion Parallel Manipulator for Nano-alignment Applications,2011 IEEE International Conference on Robotics and Automation Shanghai International Conference Center May 9-13, Shanghai, China

[8]. J.Jesus Cervantes-Sanchez n, Jose M. Rico-Martınez, SalvadorPacheco-Gutierrez, Gustavo Cerda-Villafana (2012): Static analysis of spatial parallel manipulators by means of the principle of virtual work ,International Journal of Robotics and Computer-Integrated Manufacturing vol 28, pp 385-401.

[9]. Hee-Seong Park, Chang-Hwan Choi, Sung-Hyun Kim, Byung-Suk Park (2009): Simulation of Remote Manipulator using a Virtual Engineering, IEEE International Symposium on Assembly and Manufactunng 17-20 November, Suwon, Korea

[10].RizanT. Passerone R. Macii, D. Palopoli L. Modelbased design of embedded control software for hybrid vehicles (2011): Published in Industrial Embedded Systems (SIES), 6th IEEE International Symposium. pp 75-78

[11].Karim AbdeI-Malek, Burton Paul (1998): Criteria for the Design of Manipulator Arms for a High Stiffnessto-Weight Ratio, journal of Manufacturing Systems Vol. 17/No. 3

[12].Jaydeep Roy and Louis L. Whitcomb (1999): Comparative Structural Analysis of 2-DOF SemiDirect-Drive Linkages for Robot Arms, IEEE/ASME Transactions on Mechatronics, Vol. 4, no. 1

[13].D. A. Fresonkee. Hernandez Dr. D. Tesar (1988): Deflection Prediction for Serial Manipulators, IEEE CH2555-1/88/0000/0482

[14].F. Hao, J.-P. Merlet (2005): Multi-criteria optimal design of parallel manipulators based on interval analysis, International Journal of Mechanism and Machine Theory vol $40 \mathrm{pp} 157-171$

[15].Pietro Buttolo and Blake Hannaford(1995): Pen-Based Force Display for Precision Manipulation in Virtual Environments, Proceedings of the Virtual Reality Annual International Symposium 